J. Urol. Urogynäkol. AT 2019 · 26:33-34 https://doi.org/10.1007/s41972-019-0068-0

(c) Der/die Autor(en) 2019

\section{Daniela Ulrich}

Universitätsklinik für Frauenheilkunde und Geburtshilfe, Klinische Abteilung für Gynäkologie, Medizinische Universität Graz, Graz, Österreich

\title{
Sexualität im Lebenszyklus - bei Brustkrebs
}

Sexualität ist seit 2002 laut Weltgesundheitsorganisation (WHO) als grundlegendes menschliches Bedürfnis gelistet. Die sexuelle Gesundheit ist untrennbar mit Gesundheit im Allgemeinen, Wohlbefinden und Lebensqualität verbunden. Darüber hinaus setzt sie eine positive und respektvolle Haltung zu Sexualität und sexuellen Beziehungen voraus; angenehme und sichere sexuelle Erfahrungen sollen frei von Zwang, Diskriminierung und Gewalt erlebt werden.

Die weibliche Sexualität ist ein komplexes Zusammenspiel aus körperlichen, psychischen, zwischenmenschlichen und umgebungsbedingten Elementen. Sexualität kann nur dann positiv erlebt werden, wenn jede dieser Ebenen intakt ist. Jede Frau hat ihre eigenen Lustquellen bzw. erogenen Zonen, die durch Stimulation zu einer Erregung führen können. Es sind diverse Faktoren erforderlich, damit sowohl Lust als auch Erregung empfunden werden können und ein Orgasmus im Sinne des Sexualitätszirkels nach Masters u. Johnson [1] bzw. Whipple u. BrashMcGreer [2] möglich wird. Ein intaktes Körperbild ist auch Voraussetzung für die Akzeptanz im eigenen Körper; ein gesunder Körper ist notwendig, um erogene Zonen stimulieren zu können.

Sexuelle Dysfunktion ist u. a. definiert als eine Störung im Sexualitätszirkel oder Schmerzen beim Geschlechtsverkehr und weist auch bei gesunden postmenopausalen Frauen eine Prävalenz von bis zu $50 \%$ auf [3]; bei Frauen mit Brustkrebs sogar bis zu $100 \%$ [4].

Die Wahrnehmung der eigenen Attraktivität wird durch jegliche Krebserkrankung zutiefst erschüttert, und das Selbstbild kann aus dem Gleichgewicht geraten. Heftige Gefühlsreaktionen wie
Scham oder Ekel können erlebt werden, und es ist insgesamt ein langer Weg, bis sich die Betroffenen mit ihrer Versehrtheit aussöhnen. $\mathrm{Zu}$ dem eigenen $\mathrm{Ge}$ fühlschaos können weitere Ängste hinzukommen; etwa die Angst vor der Ablehnung durch den Partner oder Veränderung der Wahrnehmung durch diesen. Jegliche Veränderungen in der Gefühlswelt führen zu Veränderungen im Lust- und Erregungskreislauf mit massiven Auswirkungen auf die Sexualität jeder betroffenen Frau.

$\mathrm{Zu}$ den häufigsten Sexualstörungen gehören eine Verminderung der Lust bzw. Erregungsfähigkeit, Orgasmusstörungen und Dyspareunie. Lustlosigkeit sowie Schmerzen beim Geschlechtsverkehr sind die häufigsten Probleme und treten bei Frauen mit Brustkrebs in einer deutlich höheren Inzidenz auf als bei Frauen ohne diese Erkrankung. Neben emotionalen können auch körperliche Faktoren die Sexualität negativ beeinflussen. Eine Narbe an der Brust kann unabhängig vom Schweregrad des operativen Eingriffes zu Sensibilitätsstörungen führen. Dies wiederum vermag den Lust-Erregungs-Zirkel ebenso zu stören. Bei größeren Operationen ist das Körperbild durch einschneidende Faktoren wie das Fehlen der Brust für weitere psychosoziale Veränderungen verantwortlich [5]. Weiters greifen neoadjuvante und adjuvante Therapien massiv in das biopsychosoziale Modell ein. Haarverlust, Schleimhautschäden, Nervenirritation, Fatigue usw. wirken sich sowohl auf körperlicher als auch auf psychischer Ebene negativ auf die Sexualität aus. Antihormonelle begleitende Therapien senken die Zirkulation von Geschlechtshormonen und erzeugen
Nebenwirkungen wie sie normalerweise nur im Wechsel erlebt werden, wie z. B. Stimmungsschwankungen, Schlafstörungen, Gelenksschmerzen bzw. Scheidentrockenheit [6]. Die verminderte Lubrikation der Scheide ist mit Brennen und Juckreiz, Dranginkontinenz oder Schmerzen beim Geschlechtsverkehr assoziiert.

Sexualstörungen dauern oft auch nach Abschluss der primären Therapie an, unabhängig von einer weiterführenden adjuvanten Therapie, und können über viele Jahre nach dem Auftreten der Krankheit fortbestehen. Die Lebensqualität dieser Frauen ist dadurch massiv beeinträchtigt. Als behandelnder Arzt ist es sehr wichtig, von Anfang an begleitende Maßnahmen zur Verhinderung von sexuellen Problemen zu empfehlen. Im Vordergrund steht die Behandlung von möglichen Nebenwirkungen, die durch die Krebstherapie verursacht werden. Außerdem ist eine rechtzeitige psychologische Betreuung ein wichtiger Eckpfeiler, um psychische und physische Schmerzen besser verarbeiten zu können und um mit der Veränderung des Körperbildes umgehen zu lernen. Lokale Maßnahmen im Bereich der Scheide sind unabdingbar, um einer Dyspareunie durch Atrophie vorzubeugen. Hier wäre z. B. die Therapie mit lokaler Hyaluronsäure empfehlenswert. Die intravaginale Lasertherapie stellt eine weitere neue Therapieoption dar, sollte allerdings unter Aufsicht bzw. im Rahmen von klinischen Studien durchgeführt werden. Lokales Östrogen ist bei Frauen ohne Brustkrebs das Mittel der Wahl, wird jedoch bei Brustkrebs sehr zurückhaltend verschrieben bzw. ist teilweise kontraindiziert. 
Schließlich ist die Aufklärung der Betroffenen sehr wichtig. Frauen sollen darauf hingewiesen werden, dass sich die Sexualität durch die Diagnose Brustkrebs verändern kann und dass entsprechende Maßnahmen notwendig sind, um das Ausmaß der sexuellen Dysfunktion möglichst gering zu halten [7]. Bei anhaltenden sexuellen Problemen über die Krankheit hinaus kann mit therapeutischer Hilfe eine neue Form der Sexualität erlernt werden. Des Weiteren ist es sehr wichtig, den Partner im Rahmen einer Paartherapie in die Behandlung mit einzubeziehen, wann immer dies erforderlich erscheint.

\section{Korrespondenzadresse}

Assoz. Prof. PD Dr. Daniela Ulrich, PhD Universitätsklinik für Frauenheilkunde und Geburtshilfe, Klinische Abteilung für Gynäkologie, Medizinische Universität Graz Auenbruggerplatz 14, $8036 \mathrm{Graz}$, Österreich daniela.ulrich@medunigraz.at

Funding. Open access funding provided by Medical University of Graz.

\section{Einhaltung ethischer Richtlinien}

Interessenkonflikt. D. Ulrich gibt an, dass kein Interessenkonflikt besteht.

Dieser Beitrag beinhaltet keine vom Autor durchgeführten Studien an Menschen oder Tieren.

Open Access. Dieser Artikel wird unter der Creative Commons Namensnennung 4.0 International Lizenz (http://creativecommons.org/licenses/by/4.0/deed. de) veröffentlicht, welche die Nutzung, Vervielfältigung, Bearbeitung, Verbreitung und Wiedergabe in jeglichem Medium und Format erlaubt, sofern Sie den/die ursprünglichen Autor(en) und die Quelle ordnungsgemäß nennen, einen Link zur Creative Commons Lizenz beifügen und angeben, ob Änderungen vorgenommen wurden.

Hinweis des Verlags. Der Verlag bleibt in Hinblick auf geografische Zuordnungen und Gebietsbezeichnungen in veröffentlichten Karten und Institutsadressen neutral.

\section{Literatur}

1. Masters WH, Johnson VE (1966) Human sexual response, 1981. Aufl. Bantam Books, Toronto, New York

2. Whipple B, Brash-McGreer K (1997) Management of female sexual dysfunction. In: Sipski ML, Alexander $\mathrm{CJ}(\mathrm{Hrsg})$ Sexual function in people with disability and chronic illness. A health professional's guide. Aspen Publishers, Gaithersburg, S 509-534

J. Urol. Urogynäkol. AT 2019 • 26:33-34 https://doi.org/10.1007/s41972-019-0068-0

(c) Der/die Autor(en) 2019

\section{Ulrich}

\section{Sexualität im Lebenszyklus - bei Brustkrebs}

Zusammenfassung

Sexualität ist ein grundlegendes menschliches Bedürfnis und ein komplexes Zusammenspiel aus körperlichen, psychischen, zwischenmenschlichen und umgebungsbedingten Elementen. Sexuelle Gesundheit basiert bei Frauen auf dem Sexualitätszirkel nach Masters u. Johnson bzw. Whipple u. Brash-McGreer. Wenn einer der 4 bzw. 5 notwendigen Parameter (Lust-, Erregungs-, Plateau-, Orgasmusund Resolutionsphase) irritiert ist, kommt es zu sexueller Dysfunktion. Sind Lustlosigkeit und Schmerzen beim Geschlechtsverkehr schon häufige Probleme bei gesunden Frauen, werden sie naturgemäß vermehrt bei Frauen mit der Diagnose Brustkrebs beobachtet. Dann sind physische und psychische Probleme ursächlich an sexueller Dysfunktion beteiligt. Es ist wichtig, Frauen von Anfang an über mögliche Komplikationen im Hinblick auf ihre sexuelle Gesundheit hinzuweisen und begleitende Maßnahmen einzuleiten, um das Ausmaß möglichst gering zu halten. Bei Beschwerden, die über die Dauer der Therapie des Tumors hinausgehen, soll auf jeden Fall sexualmedizinische Hilfe angeboten werden.

\section{Schlüsselwörter}

Sexualität · Brustkrebs · Lustlosigkeit Schmerzen · Sexuelle Dysfunktion

\section{Sexuality in the life cycle-after breast cancer}

Abstract

Sexuality is a basic human need and is a complex interplay of physical, mental, interpersonal and environmental elements. Sexual health is based on the sexual response cyrcle according to Masters and Johnson or Whipple \& Brash-McGreer. If one of the five necessary parameters for a normal sexual life is unbalanced, sexual dysfunction occurs. Hyposexual desire and pain during intercourse are common problems in healthy women and affects even more women with breast cancer. Physical and mental problems are causally involved in female sexual dysfunction. It is important to inform women about possible complications during and after treatment in regard to sexual function. Accompanying measures, sexualmedical help in particular, should be offered to minimize the severity of sexual dysfunction when the complaints last beyond the duration of the tumor therapy.

\section{Keywords}

Sexuality - Breast cancer - Hyposexual desire Pain - Sexual dysfunction
3. McCabe MP et al (2016) Incidence and prevalence of sexual dysfunction in women and men: $A$ consensus statement from the fourth international consultation on sexual medicine 2015. J Sex Med 13(2):144-152

4. Jing L et al (2019) Incidence and severity of sexual dysfunction among women with breast cancer: A meta-analysis based on female sexual function index. Support Care Cancer. https://doi.org/10. 1007/s00520-019-04667-7

5. Aerts $L$ et al (2014) Sexual functioning in women after mastectomy versus breast conserving therapy for early-stage breast cancer: A prospective controlled study. Breast 23(5):629-636

6. Gambardella A et al (2018) Sexual function and sex hormones in breast cancer patients. Endocrine 60(3):510-515

7. Hummel SB et al (2017) Factors associated with specific diagnostic and statistical manual of mental disorders, fourth edition sexual dysfunctions in breast cancer survivors: A study of patients and their partners. J Sex Med 14(10):1248-1259 